\title{
Simulation of double diffusive convection in fluid-saturated porous media by lattice Boltzmann method
}

\author{
Sheng Chen ${ }^{* 1,2,3}$ Bo Yang ${ }^{1}$ Chuguang Zheng ${ }^{1}$ \\ 1. State Key Laboratory of Coal Combustion, Huazhong University of Science and \\ Technology, Wuhan 430074, China \\ 2. Institute for Modelling and Simulation in Fluodynamics, Nanoscience and \\ Industrial Mathematics "Gregorio Millán Barbany", Universidad Carlos III de \\ Madrid, Leganes 28911, Spain \\ 3. Faculty of Engineering, The University of Nottingham, University Park, \\ Nottingham NG7 2RD, UK \\ * Corresponding author. Faculty of Engineering, University of Nottingham. E-mail \\ address: shengchen.hust@gmail.com
}

\begin{abstract}
The research on double diffusive convection in porous media is important to deepen our insights into sustainable development and environment protection. A lattice Boltzmann (LB) model for REV (representative elementary volume) scale simulation of double diffusive convection in fluid-saturated porous media is proposed in the present work. It can work well not only for porous media with uniform porosity but also for non-uniform porous media. Several benchmark tests are adopted to validate its capability. The good agreement with previous publications demonstrates its applicability. It can provide an alternative numerical tool for modelling complex heat and mass transfer in fluid-saturated porous media beyond double diffusive convection, such as heat and moisture transfer in multi-layer building materials.
\end{abstract}

Key words: Lattice Boltzmann method; heat and mass transfer; porous media; double diffusive convection; non-uniform porosity

\section{Introduction}

Double diffusive convection [1,2] in fluid-saturated porous media is commonly found in nature, as well as in industry, such as pollution spreading in soil [3] and solute cycles in solar collectors [4]. So far numerical methods have been 
popularly adopted to deepen our insights into the fundamentals of heat and mass transfer within fluid-saturated porous media. Bejan et al. [5] and Pop et al. [6] perhaps are the pioneers in this field and they reviewed the progress of the early stage of this field. As the public's concern on sustainable development and environment protection has been soaring, research on double diffusive convection in fluid-saturated porous media has received increasing attention again. The effects of the Rayleigh and Darcy numbers on double diffusive convection in a porous cavity were discussed in Ref.[7]. Through their numerical simulation one can observe that the behaviors of buoyant flow in a cavity filled by porous media were quite different from its non-porous counterpart [8]. Mondal and Sibanda [9] conducted a numerical study on influences of buoyancy ratio on unsteady double diffusive natural convection in a porous cavity. The authors claimed the patterns of heat and mass transfer in porous media would change significantly. Double diffusive convection of nanofluid in a porous enclosure was simulated in Ref.[10]. It was reported that heat transfer was reduced by increasing the bulk volume fraction of nanoparticles. Turbulent double diffusive convection in porous media was also investigated numerically[11]. The work implied new numerical models are desired to deeply reveal the fundamentals of double diffusive convection in fluid-saturated porous media. The amount of publications on this topic is so huge and only a few can be cited here. In almost all research on this topic, the REV (representative elementary volume) scale mathematic description is adopted to model double diffusive convection in fluid-saturated porous media as a REV scale model can not only provide acceptable accuracy for engineering applications but also save computational cost for industrial-scale simulation $[12,13]$.

During the past three decades, the lattice Boltzmann (LB) method has attracted increasing attention as an alternative simulation tool[14]. Originally, the LB method was adopted as a powerful tool for pore-scale modelling of porous media [15-17]. The first REV scale LB model for isothermal fluidsaturated porous media was proposed by Guo and Zhao [18]. Soon, the same authors designed a thermal LB model for simulation of heat transfer in porous media [19]. Due to its simplicity and reliability, their REV scale LB model has been widely used for research on heat transfer in porous media, such as phase change in porous media [20,21], natural convection in porous media [22] and convective heat transfer in a channel partially filled by porous media [23]. In order to improve the numerical stability of Guo's model, which is based on the so-called single-relaxation-time (SRT) approximation, a multiple-relaxationtime (MRT) counterpart has been constructed by Liu et al. [20,24]. Recently, several LB models for non-equilibrium heat transfer in fluid-saturated porous media were published $[25,26]$. Meanwhile, there have been numerous publications on modelling double diffusive convection by the LB method [27-33]. However, the effort to extend the LB method to model double diffusive convection in fluid-saturated porous media at the REV scale is absent yet. 
As the macroscopic governing equation for concentration transfer (e.g. Eq.(4)) is a so-called advection-diffusion equation which is similar with that for heat transfer (e.g. Eq.(3)), it seems straightforward to construct a LB model for double diffusive convection in fluid-saturated porous media along the line proposed by Guo et al. [19]. Unfortunately, as shown below, such way will suffer a serious shortcoming that the porosity in the whole investigated domain should be uniform. However, for many realistic scenarios, the porosity is non-uniform [4,34-38]. In addition, by such way the effective mass diffusivity will depend on the local porosity, which is non-physical.

The purpose of the present work is to bridge the above gap. The rest of the present paper is organized as follows. In Section2, a LB model for double diffusive convection in fluid-saturated porous media is presented, which can treat non-uniform porosity easily. An analysis is presented to show the drawback to extend the available LB models for simulation of double diffusive convection in fluid-saturated porous media. Numerical validation for the present model is conducted in Section 3, followed by a conclusion on this work.

\section{LB model for double diffusive convection in fluid-saturated porous media}

In the present work, we take a SRT-based LB model as an example to show how to construct a LB model for double diffusive convection in fluid-saturated porous media. It is straightforward to establish its MRT-based counterpart in the same way, for example, following the MRT-based porous media model proposed by Liu et al. [20,24].

2.1 Macroscopic governing equations for double diffusive convection in fluidsaturated porous media

The popularly used macroscopic governing equations for double diffusive convection in fluid-saturated porous media read $[4,39,40]$ :

$$
\begin{gathered}
\nabla_{\alpha} u_{\alpha}=0, \\
\partial_{t} u_{\alpha}+u_{\alpha} \nabla_{\alpha} \frac{u_{\beta}}{\epsilon}=\nabla_{\alpha} \epsilon p+\nabla_{\alpha} \nu_{e} \nabla_{\alpha} u_{\alpha}+F_{\alpha}, \\
\sigma \partial_{t} T+\nabla_{\alpha} T u_{\alpha}=\nabla_{\alpha} \kappa_{e} \nabla_{\alpha} T, \\
\epsilon \partial_{t} \phi+\nabla_{\alpha} \phi u_{\alpha}=\nabla_{\alpha} D_{e} \nabla_{\alpha} \phi .
\end{gathered}
$$

where $u_{\alpha}\left(u_{\beta}\right), T$ and $\phi$ are the volume-averaged velocity, temperature of fluid and concentration in the saturated porous media, respectively. The parameter 
$\sigma$ denotes the ratio of heat capacitance (the product of density and specific heat capacity) between saturated porous media and saturating fluid and $\epsilon$ is the porosity of the porous media. In addition, $\nu_{e}, \kappa_{e}$ and $D_{e}$ are the effective kinematic viscosity, effective thermal diffusivity and effective mass diffusivity, respectively. Usually, $\nu_{e}=\nu$ where $\nu$ is the kinematic viscosity of saturating fluid.

The force term $F_{\alpha}$ in Eq.(2) reads:

$$
F_{\alpha}=-\frac{\epsilon \nu}{K} u_{\alpha}-\frac{1.75}{\sqrt{150 \epsilon K}}\left|u_{\alpha}\right| u_{\alpha}+g_{\alpha} \beta_{T}\left(T-T_{0}\right)+g_{\alpha} \beta_{\phi}\left(\phi-\phi_{0}\right) .
$$

where $g_{\alpha}$ is the gravity acceleration and $K$ is the permeability of porous media. $\beta_{T}$ and $\beta_{\phi}$ are the coefficient of thermal expansion and of concentration expansion, respectively. In addition, $T_{0}$ and $\phi_{0}$ are the reference values of temperature and concentration, respectively. In our simulation we set $T_{0}=\phi_{0}=0$. The former two terms of $F_{\alpha}$ represent the body force due to the presence of a porous medium and the last two denote the buoyant force due to temperature and concentration difference.

The thermal Rayleigh number $\left(R a_{T}\right)$ is defined as

$$
R a_{T}=g_{\alpha} \beta_{T}\left(T-T_{0}\right) / \nu \kappa
$$

and the solute Rayleigh number $\left(R a_{\phi}\right)$ is defined as

$$
R a_{\phi}=g_{\alpha} \beta_{\phi}\left(\phi-\phi_{0}\right) / \nu \kappa
$$

where $\kappa$ is the thermal diffusivity of fluid. Accordingly, the buoyancy ratio is defined as

$$
N=R a_{T} / R a_{\phi}
$$

The dimensionless formula of the above macroscopic governing equations read:

$$
\begin{gathered}
\nabla_{\alpha} \bar{u}_{\alpha}=0 \\
\partial_{t} \bar{u}_{\alpha}+\bar{u}_{\alpha} \nabla_{\alpha} \frac{\bar{u}_{\beta}}{\epsilon}=\nabla_{\alpha} \epsilon \bar{p}+\frac{P r}{\sqrt{R a_{T}}} \nabla_{\alpha} \nabla_{\alpha} \bar{u}_{\alpha}+\bar{F}_{\alpha}, \\
\sigma \partial_{t} \bar{T}+\nabla_{\alpha} \bar{T}_{\alpha}=\frac{1}{\sqrt{R a_{T}}} \nabla_{\alpha} \nabla_{\alpha} \bar{T} \\
\epsilon \partial_{t} \bar{\phi}+\nabla_{\alpha} \bar{\phi}_{\alpha}=\frac{L e}{\sqrt{R a_{T}}} \nabla_{\alpha} \nabla_{\alpha} \bar{\phi} . \\
\bar{F}_{\alpha}=-\frac{\epsilon \operatorname{Pr}}{\sqrt{R a_{T}} D a} \bar{u}_{\alpha}-\frac{1.75}{\sqrt{150 \epsilon D a}}\left|\bar{u}_{\alpha}\right| \bar{u}_{\alpha}+\operatorname{Pr}(\bar{T}+N \bar{\phi}) \frac{g_{\alpha}}{\left|g_{\alpha}\right|} .
\end{gathered}
$$

The overbar in the above equations indicates dimensionless variables. The normalization process please refer to Ref.[32]. In Eqs.(9)-(13), Pr, Da and Le 
are the Prandtl number, Darcy number and Lewis number, respectively.

\subsection{Evolving equation}

As the governing equations for flow and temperature field, namely Eqs.(1)(3), can be solved by Guo's [19] or Guo's-like [20,24] LB models, here we only present the LB model for the concentration field (Eq.(4)).

The SRT-based LB evolving equation for concentration field reads:

$$
g_{j}\left(x_{\alpha}+c e_{j \alpha} \Delta t, t+\Delta t\right)-g_{j}\left(x_{\alpha}, t\right)=-\tau_{\phi}^{-1}\left[g_{j}\left(x_{\alpha}, t\right)-g_{j}^{(e q)}\left(x_{\alpha}, t\right)\right] .
$$

In Eq.(14) $\tau_{\phi}$ is the dimensionless relaxation time for the pseudo-particle distribution $g_{j}\left(x_{\alpha}, t\right)$ at space $x_{\alpha}$ and instant $t . c e_{j \alpha}$ denotes the discrete velocities and $\Delta t$ means the time interval in the LB evolving equation.

If we follow the way proposed by Guo et al. [19], the equilibrium distribution function $g_{j}^{(e q)}$ in Eq.(14) will read:

$$
g_{j}^{(e q)}=\omega_{j} \phi\left(\epsilon+\frac{c e_{j \alpha} u_{\alpha}}{c_{s}^{2}}\right)
$$

Unfortunately, as shown below, if the above equilibrium distribution function Eq.(15) is adopted, we have to assume the porosity $\epsilon$ is a constant over the whole domain and the effective mass diffusivity depends on the local porosity.

In order to remedy these shortcomings, in the present work the equilibrium distribution function $g_{j}^{(e q)}$ in Eq.(14) is defined as:

$$
g_{j}^{(e q)}=\left\{\begin{array}{l}
\phi\left(\epsilon-\epsilon_{0}\right)+\omega_{j} \phi\left(\epsilon_{0}+\frac{c e_{j \alpha} u_{\alpha}}{c_{s}^{2}}\right), \quad j=0 \\
\omega_{j} \phi\left(\epsilon_{0}+\frac{c e_{j \alpha} u_{\alpha}}{c_{s}^{2}}\right), \quad j \neq 0
\end{array}\right.
$$

where $\omega_{j}$ represents the weight coefficient. $\epsilon_{0}$ is a reference value of porosity so $\epsilon_{0}$ is a constant within the whole investigated domain. In order to guarantee numerical stability, generally we chose the minimum value of porosity in the investigated domain as $\epsilon_{0}$. The parameter $c_{s}$ satisfies $c_{s}^{2} \delta_{\alpha \beta}=\sum_{j} \omega_{j} c^{2} e_{j \alpha} e_{j \beta}$ [41].

In the present work, the same as Ref. [19], a D2Q5 lattice for two-dimensional problems is used for modelling concentration field. For three-dimensional investigated domains, one can invoke a D3Q7 lattice [42]. Such choice can save computational cost efficiently, which is crucial for industrial-level simulation, as explained in our previous work [41]. 
The concentration $\phi$ is obtained by

$$
\phi=\frac{\sum_{j} g_{j}}{\epsilon} .
$$

and the effective mass diffusivity $D_{e}$ is given by

$$
D_{e}=\epsilon_{0}\left(\tau_{\phi}-1 / 2\right) c_{s}^{2} \Delta t
$$

According to Eq.(18), it is clear that in the present model the effective mass diffusivity $D_{e}$ is independent of the local porosity $\epsilon$ and there is no restriction that $\epsilon$ should be uniform.

\subsection{Multiscale expansion and recovered macroscopic equation}

Equation (14) can be expanded in Taylor series as [14]

$$
\Delta t\left(\partial_{t}+c e_{j \alpha} \nabla_{\alpha}\right) g_{j}+\frac{\Delta t^{2}}{2}\left(\partial_{t}+c e_{j \alpha} \nabla_{\alpha}\right)^{2} g_{j}+\frac{1}{\tau_{\phi}}\left[g_{j}-g_{j}^{(e q)}\right]=O\left(\Delta t^{3}\right) .
$$

Introducing the multiscale expansion $\partial_{t}=\varepsilon \partial_{t 1}+\varepsilon^{2} \partial_{t 2}, \nabla_{\alpha}=\varepsilon \nabla_{\alpha 1}$ and $g_{j}=$ $g_{j}^{(e q)}+\varepsilon g_{j}^{(1)}+\varepsilon^{2} g_{j}^{(2)}+O\left(\varepsilon^{3}\right)$, where $\varepsilon$ is a slight quantity [19], we can sort Eq. (19) in terms of $\varepsilon$ and $\varepsilon^{2}$ as

$$
\begin{gathered}
\left(\partial_{t 1}+c e_{j \alpha} \nabla_{\alpha 1}\right) g_{j}^{(e q)}=-\frac{g_{j}^{(1)}}{\Delta t \tau_{\phi}}+O(\varepsilon) . \\
\partial_{t 2} g_{j}^{(e q)}+\left(\partial_{t 1}+c e_{j \alpha} \nabla_{\alpha 1}\right)\left[\left(1-\frac{1}{2 \tau_{\phi}}\right) g_{j}^{(1)}\right]=-\frac{g_{j}^{(2)}}{\Delta t \tau_{\phi}}+O\left(\varepsilon^{2}\right) .
\end{gathered}
$$

With the symmetry properties of the lattice $\sum_{j} \omega_{j} c e_{j \alpha}=0, \sum_{j} \omega_{j} c e_{j \alpha} c e_{j \beta}=$ $c_{s}^{2} \delta_{\alpha \beta}$ and the equilibrium distribution function $g_{j}^{(e q)}$ defined by Eq.(16), we can obtain

$$
\begin{gathered}
\sum_{j} g_{j}^{(e q)}=\epsilon \phi, \\
\sum_{j} c e_{j \alpha} g_{j}^{(e q)}=\phi u_{\alpha}, \\
\sum_{j} c e_{j \alpha} c e_{j \beta} g_{j}^{(e q)}=\epsilon_{0} \phi c_{s}^{2} \delta_{\alpha \beta} .
\end{gathered}
$$

With the aid of Eqs.(22)-(24), as well as $\sum_{j} g_{j}^{(1)}=\sum_{j} g_{j}^{(2)}=0$, the summation 
of Eqs.(20)-(21) over the discrete direction $e_{j \alpha}$ reads

$$
\begin{gathered}
\partial_{t 1} \epsilon \phi+\nabla_{\alpha 1} \phi u_{\alpha}=0+O(\varepsilon), \\
\partial_{t 2} \epsilon \phi+\nabla_{\alpha 1}\left[c_{s}^{2}\left(\frac{1}{2}-\tau_{\phi}\right) \Delta t \nabla_{\alpha 1} \epsilon_{0} \phi\right]=0+O\left(\varepsilon^{2}\right) .
\end{gathered}
$$

Because $\epsilon_{0}$ is a constant across the whole investigated domain, $\nabla_{\alpha 1} \epsilon_{0} \phi=$ $\epsilon_{0} \nabla_{\alpha 1} \phi$. Accordingly Eq.(26) can be re-written as

$$
\partial_{t 2} \epsilon \phi+\nabla_{\alpha 1}\left[\epsilon_{0} c_{s}^{2}\left(\frac{1}{2}-\tau_{\phi}\right) \Delta t \nabla_{\alpha 1} \phi\right]=0+O\left(\varepsilon^{2}\right) .
$$

Combining Eqs.(25) and (27), we can obtain the final recovered macroscopic governing equation for the concentration field

$$
\partial_{t} \epsilon \phi+\nabla_{\alpha} \phi u_{\alpha}=\nabla_{\alpha} D_{e} \nabla_{\alpha} \phi+O\left(\varepsilon^{2}\right) .
$$

where $D_{e}=\epsilon_{0} c_{s}^{2}\left(\tau_{\phi}-\frac{1}{2}\right) \Delta t$. It is obvious that Eq.(28) can match Eq.(4) exactly, no matter whether $\epsilon$ is non-uniform.

However, if Guo's-like equilibrium distribution function Eq.(15) is adopted, the second moment of the equilibrium distribution $g_{j}^{(e q)}$ will depend on $\epsilon$, namely

$$
\sum_{j} c e_{j \alpha} c e_{j \beta} g_{j}^{(e q)}=\epsilon \phi c_{s}^{2} \delta_{\alpha \beta} .
$$

Please pay attention to the difference between Eq.(24) and Eq.(29). Accordingly, through the multiscale expansion, it will generate

$$
\partial_{t 2} \epsilon \phi+\nabla_{\alpha 1}\left[c_{s}^{2}\left(\frac{1}{2}-\tau_{\phi}\right) \Delta t \nabla_{\alpha 1} \epsilon \phi\right]=0+O\left(\varepsilon^{2}\right) .
$$

In Eq.(30), to move $\epsilon$ outside from the spatial gradient operator $\nabla_{\alpha 1}$ (i.e. $\left.\nabla_{\alpha 1} \epsilon \phi=\epsilon \nabla_{\alpha 1} \phi\right)$, one has to assume $\epsilon$ to be uniform, namely $\nabla_{\alpha 1} \epsilon=0$. Furthermore, the effective mass diffusivity $D_{e}=\epsilon c_{s}^{2}\left(\tau_{\phi}-\frac{1}{2}\right) \Delta t$, which is nonphysical as now $D_{e}$ depends on the local porosity $\epsilon$.

\section{Numerical validation}

In order to validate the present model, two types of benchmark tests are adopted. The first one is double diffusive convection in a rectangular enclosure filled by uniform fluid-saturated porous media and the second one is double diffusive convection in a bi-layered heterogenous porous cavity. 
3.1 Double diffusive convection in a rectangular enclosure filled by uniform fluid-saturated porous media

The configuration and boundary conditions of the uniform porous enclosure are illustrated by Fig. 1, where $H$ and $L$ are the height and width of the enclosure, respectively.

Firstly, we set $H / L=2, \epsilon=0.999, \sigma=1$, the Rayleigh number $R a_{T}=10^{5}$, the Darcy number $D a=10^{6}$, the Prandtl number $\operatorname{Pr}=1$ and the Lewis number $L e=2$. The buoyancy ratio $N=-0.8$ and -1.3 , respectively. As $\epsilon$ is very close to unity and $D a \gg 1$, the convective flow in the porous enclosure should be almost the same as its counterpart without porous pack [43]. A grid resolution $100 \times 200$ is adopted, as shown in our previous work [43] such grid resolution is fine enough to reproduce grid-independent solutions. Figures 2-3 plot the streamlines, isotherms and isoconcentrations for different $N$. When $N<-1$, the flow is primarily dominated by thermal buoyancy effects, and a large central clockwise thermal recirculation is predicted with horizontally non-uniform isotherms in the core region within the enclosure. Furthermore, the concentration contours are distorted in the core of the enclosure with a stable stratification in the vertical direction except near the insulated walls of the enclosure. A stagnant zone in the corners of the enclosure is also observed. In contrast, for $N>-1$ the flow is mainly dominated by compositional buoyancy effects. For example, when $N=-1.3$, a counterclockwise compositional recirculation exists in the core region of the enclosure along with two clockwise thermal recirculations occurring near the top right and bottom left corners of the enclosure. The contours for temperature and concentration are almost parallel to each other within the center of the enclosure away from the walls. The observations agree well with those reported by Ref.[43] (c.f. Figs. 2-3 in Ref.[43]). Table 1 lists the calculated average Nusselt $(N u)$ and Sherwood $(S h)$ number on the hot wall, together with those published by Ref.[43]. The quantitative comparison demonstrates the reliability of the present model to predict double diffusive convection in fluid-saturated porous media with different $N$. In the present work the average Nusselt number $N u$ is defined as

$$
N u=-\frac{1}{L} \int_{0}^{L} \frac{\partial T}{\partial x} d y
$$

where $L$ is the height of the investigated domain.

Then we set $H / L=1, \epsilon=0.8, \sigma=1$, the modified Rayleigh number $R a_{M}=D a \times R a_{T}=100, \operatorname{Pr}=0.71, N=1, L e=10$ while $10^{-1} \leq D a \leq 10^{-3}$. A grid resolution $100 \times 100$ is employed. The streamlines, isotherms and isoconcentrations for various $D a$ are depicted by Figs.4-6. A large clockwise recirculation is formed in the domain. With $D a$ increasing, the recirculation is stretched and convective heat transfer, replacing conductive heat transfer, 
becomes the predominant heat transfer mechanism. As $L e \gg 1$, the isoconcentrations are distorted more significantly than the isotherms. The observations are consistent with that reported in Ref.[44] (c.f. Fig.3 in Ref.[44]). Table 2-3 present the calculated $N u$ and $S h$, compared with those listed in Ref.[44]. The numerical predictions by the present model agree well with previous data in Ref.[44], which shows the capability of the present model for simulation of double diffusive convection within fluid-saturated porous media with different Da. Although in Ref.[44] the investigated the domain is a cubic domain, we find that for laminar double diffusive convection our two-dimensional results are identical to their three-dimensional ones.

\subsection{Double diffusive convection in a bi-layered heterogenous porous cavity}

Double diffusive convection in a bi-layered heterogenous porous cavity, which was investigated in Ref.[45], is adopted here to validate the reliability of the present model for modelling heat and mass transfer in non-uniform porous media where porosity is not identical. The configuration of the bi-layered heterogenous porous cavity is illustrated by Fig. 7. The parameters used in the present work read: $R a_{T}=10^{6}, \operatorname{Pr}=7, N=2, \sigma=1$ and $L e=1,10$. The Darcy number and the porosity of the bi-layers are $D a_{1}=10^{-4}, \epsilon_{1}=0.4$ (the lower layer) and $D a_{2}=10^{-5}, \epsilon_{2}=0.5$ (the upper layer). A grid resolution $100 \times 100$ is adopted.

Figures 8-9 plot the streamlines, isotherms and isoconcentrations. The flow resistance is smaller in the lower layer as its permeability is larger than the upper layer. Consequently, the streamlines within the lower layer are elliptical extended. As the flow in the upper layer moves relatively slowly, the dominant heat transfer mechanism is conductive heat transfer. Accordingly, the isotherms within the upper layer are nearly vertical and parallel with each other. In the lower layer, benefitting from the smaller flow resistance, convective heat transfer becomes predominant and accordingly the isotherms are distorted obviously. When $L e=1$, the distributions of isotherms and of isoconcentrations are identical. With Le increasing, the isoconcentrations are deformed significantly, especially in the lower layer. A solute boundary layer is formed in the vicinity of the left side of the lower porous layer. The isoconcentrations are confined near the active walls of the cavity, which attributes to the high $L e$ and $N$ [45]. These observations agree with those reported in Ref.[45]. Table 4 lists the calculated $N u$ and $S h$, compared with those in Ref.[45] (please refer to Fig. 9 in Ref.[44]). The quantitative comparison demonstrates the applicability of the present model in Ref.[45] for simulation of double diffusive convection in heterogenous porous media. However, if Guo's-like equilibrium distribution function Eq.(15) is adopted, the numerical results will diverge. 


\section{Conclusion}

Double diffusive convection in fluid-saturated porous media is an important topic in many disciplines. So far numerical approaches have become an indispensable tool to deepen our insights into its fundamentals. New numerical techniques are desired to solve the problems more efficiently. In the present work, a LB approach is proposed for this purpose. It can work well not only for uniform porous media but also for non-uniform porous media as a new type of equilibrium distribution function is proposed. Furthermore, an analysis is given to explain why we can not establish the present model following the line proposed in the available publications.

Although in the present study we only take a SRT LB model as an example to show how to model double diffusive convection in fluid-saturated porous media, the extension to its MRT counterpart is straightforward as a MRT model has better numerical stability for cases with high Le. It will be considered in our future work.

\section{Acknowledgments}

This work has received funding from the Universidad Carlos III de Madrid, the European Unions Seventh Framework Programme for research, technological development and demonstration under grant agreement No. 600371, el Ministerio de Economa y Competitividad (COFUND2014-51509), el Ministerio de Educacin, cultura y Deporte (CEI-15-17) and Banco Santander. S. Chen would also acknowledge the support from the British Newton Alumni Fellowship Scheme.

\section{References}

[1] Huppert HE, Turner JS. Double-diffusive convection. Journal of Fluid Mechanics 1981;106:299-329

[2] Turner JS. Double-diffusive phenomena. Annual Review of Fluid Mechanics 1974;6: 37-54.

[3] Ragui K, Boutra A, Benkahla YK. On the validity of a numerical model predicting heat and mass transfer in porous square cavities with a bottom thermal and solute source: case of pollutants spreading and fuel leaks. Mechanics \& Industry $2016 ; 17: 311-320$ 
[4] Hadidi N, Ould-Amer Y, Bennacer R. Bi-layered and inclined porous collector: Optimum heat and mass transfer. Energy 2013;51: 422-430

[5] Nield DA, Bejan A. Convection in Porous Media. 1992 Springer, New York.

[6] Ingham DB, Pop I. Transport Phenomena in Porous Media. 2005 Elsevier, Oxford.

[7] Goyeau B, Songbe JP, Gobin D. Numerical study of double-diffusive natural convection in a porous cavity using the Darcy-Brinkman formulation. International Journal of Heat and Mass Transfer 1996;39: 1363-1378.

[8] Beghein C, Haghighat F, Allard F. Numerical study of double-diffusive natural convection in a square cavity. International Journal of Heat and Mass Transfer 1992;35: 833-846

[9] Mondal S, Sibanda P. Effects of buoyancy ratio on unsteady double-diffusive natural convection in a cavity filled with porous medium with non-uniform boundary conditions. International Journal of Heat and Mass Transfer 2015; $85: 401-413$

[10] Dastmalchi M, Sheikhzadeh GA, Arani AAA. Double-diffusive natural convective in a porous square enclosure filled with nanofluid. International Journal of Thermal Sciences 2015; 95: 88-98

[11] de Lemos MJS. Analysis of turbulent double-diffusive free convection in porous media using the two-energy equation model. International Communications in Heat and Mass Transfer 2014;52: 132-139

[12] Bear J. Dynamics of Fluids in Porous Media. 2013 Elsevier, New York.

[13] Kanit T, Forest S, Galliet I, Mounoury V, Jeulin D. Determination of the size of the representative volume element for random composites: statistical and numerical approach. International Journal of Solids and Structures 2003;40: $3647-3679$

[14] Succi S. The lattice Boltzmann equation for fluid dynamics and beyond. Oxford: Oxford university press;2001.

[15] Kang Q, Zhang D, Chen S, He X. Lattice Boltzmann simulation of chemical dissolution in porous media. Physical Review E 2002;65: 036318/1-036318/8

[16] Kuwata Y, Suga K. Large eddy simulations of pore-scale turbulent flows in porous media by the lattice Boltzmann method. International Journal of Heat and Fluid Flow 2015;55:143-157

[17] Grucelski A, Pozorski J. Lattice Boltzmann simulations of heat transfer in flow past a cylinder and in simple porous media. International Journal of Heat and Mass Transfer 2015;86: 139-148

[18] Guo Z, Zhao TS. Lattice Boltzmann model for incompressible flows through porous media. Physical Review E 2002;66: 036304/1-036304/9 
[19] Guo Z, Zhao TS. A lattice Boltzmann model for convection heat transfer in porous media. Numerical Heat Transfer, Part B 2005; 47: 157-177

[20] Liu Q, He YL. Double multiple-relaxation-time lattice Boltzmann model for solid-liquid phase change with natural convection in porous media. Physica A 2015;438:94-106

[21] Gao D, Chen Z. Lattice Boltzmann simulation of natural convection dominated melting in a rectangular cavity filled with porous media. International Journal of Thermal Science 2011;50: 493-501.

[22] Seta T, Takegoshi E, Okui K. Lattice Boltzmann simulation of natural convection in porous media. Mathematics and Computers in Simulation 2006;72: 195-200

[23] Shokouhmand H, Jam F, Salimpour MR. Simulation of laminar flow and convective heat transfer in conduits filled with porous media using lattice Boltzmann method. International Communications in Heat and Mass Transfer 2009;36 : 378-384.

[24] Liu Q, He YL, Li Q, Tao WQ. A multiple-relaxation-time lattice Boltzmann model for convection heat transfer in porous media. International Journal of Heat and Mass Transfer 2014;73:761-775

[25] Gao D, Chen Z, Chen L. A thermal lattice Boltzmann model for natural convection in porous media under local thermal non-equilibrium conditions. International Journal of Heat and Mass Transfer 2014;70:979-989

[26] Wang L, Zeng Z, Zhang L, Xie H, Liang G, Lu Y. A lattice Boltzmann model for thermal flows through porous media. Applied Thermal Engineering 2016;108:6675 .

[27] Ren Q, Chan CL. Numerical study of double-diffusive convection in a vertical cavity with Soret and Dufour effects by lattice Boltzmann method on GPU. International Journal of Heat and Mass Transfer 2016;93:538-553

[28] Xu H, Li B, Chen J, Lou Q, Yan Z, Yang M. Lattice BGK simulations of the double diffusive mixed convection in an enclosure with different outlet locations. International Journal of Heat and Mass Transfer 2016;92:158-172

[29] Chen S, Yang B, Luo KH, Xiong X, Zheng C. Double diffusion natural convection in a square cavity filled with nanofluid. International Journal of Heat and Mass Transfer 2016;95:1070-1083

[30] Bettaibi S, Kuznik F, Sediki E. Hybrid LBM-MRT model coupled with finite difference method for double-diffusive mixed convection in rectangular enclosure with insulated moving lid. Physica A 2016;444:311-326

[31] Moufekkir F, Moussaoui MA, Mezrhab A, Bouzidi M, Laraqi N. Study of double-diffusive natural convection and radiation in an inclined cavity using lattice Boltzmann method. International Journal of Thermal Sciences 2013;63:65-86 
[32] Chen S, Liu H, Zheng C. Numerical study of turbulent double-diffusive natural convection in a square cavity by LES-based lattice Boltzmann model. International Journal of Heat and Mass Transfer 2012;55: 4862-4870

[33] Verhaeghe F, Blanpain B, Wollants P. Lattice Boltzmann method for doublediffusive natural convection. Physical Review E 2007;75:046705/1-046705/18

[34] Nithiarasu P, Seetharamu KN, Sundararajan T. Natural convective heat transfer in a fluid saturated variable porosity medium. International Journal of Heat and Mass Transfer 1997;40:3955-3967

[35] Clement TP, Hooker BS, Skeen RS. Macroscopic Models for Predicting Changes in Saturated Porous Media Properties Caused by Microbial Growth. Groundwater 1996;34: 934-942

[36] Wu H, Fan J. Study of heat and moisture transfer within multi-layer clothing assemblies consisting of different types of battings. International Journal of Thermal Sciences 2008;47: 641-647

[37] Qin M, Belarbi R, Ait-Mokhtar A, Nilsson L. Coupled heat and moisture transfer in multi-layer building materials. Construction and Building Materials 2009;23: 967-975

[38] Akbal S, Baytas F. Effects of non-uniform porosity on double diffusive natural convection in a porous cavity with partially permeable wall. International Journal of Thermal Sciences 2008;47: 875-885

[39] Nithiarasu P, Seetharamu KN, Sundararajan T. Double-diffusive natural convection in an enclosure filled with fluid-saturated porous medium: a generalized non-Darcy approach. Numerical Heat Transfer, Part A 1996;30:413426

[40] Saberi A, Nikbakhti R. Numerical investigation of double diffusive natural convection in a rectangular porous enclosure with partially active thermal walls. Journal of Porous Media 2016;19:259-275

[41] Chen S, Liu Z, Zhang C, He Z, Tian Z, Shi B, Zheng C. A novel coupled lattice Boltzmann model for low Mach number combustion simulation. Applied Mathematics and Computation 2007;193 : 266-284.

[42] Chen S, Yang B, Zheng C. A lattice Boltzmann model for heat transfer in heterogeneous media. International Journal of Heat and Mass Transfer 2016;102: 637-644

[43] Chen S, Tolke J, Krafczyk M. Numerical investigation of double-diffusive (natural) convection in vertical annuluses with opposing temperature and concentration gradients. International Journal of Heat and Fluid Flow 2010;31:217-226

[44] Kramer J, Jecl R, Ravnik J, Skerget L. Double-diffusive natural convection within a $3 \mathrm{D}$ porous enclosure, using the boundary element method. 8th International Conference on Heat Transfer, Fluid Mechanics 
and Thermodynamics 2011 Pointe Aux Piments, Mauritius http : //www.repository.up.ac.za/handle/2263/41781

[45] Hadidi N, Bennacer R, Ould-Amer Y. Two-dimensional thermosolutal natural convective heat and mass transfer in a bi-layered and inclined porous enclosure. Energy 2015;93: 2582-2592 


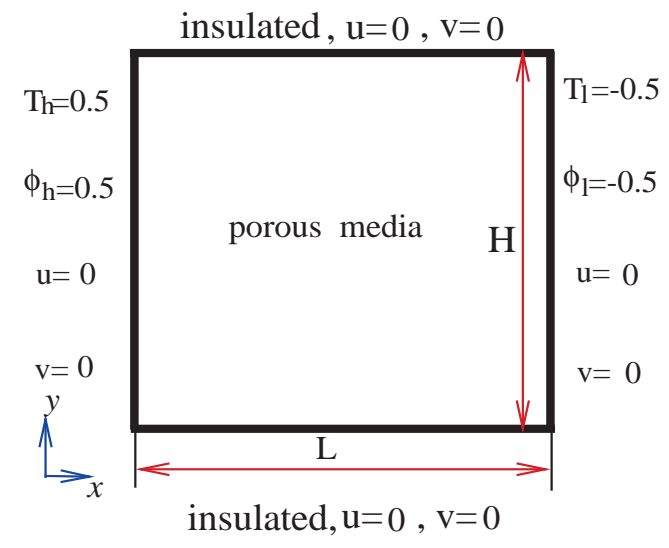

Fig. 1. Schematic configuration of the uniform porous enclosure. 


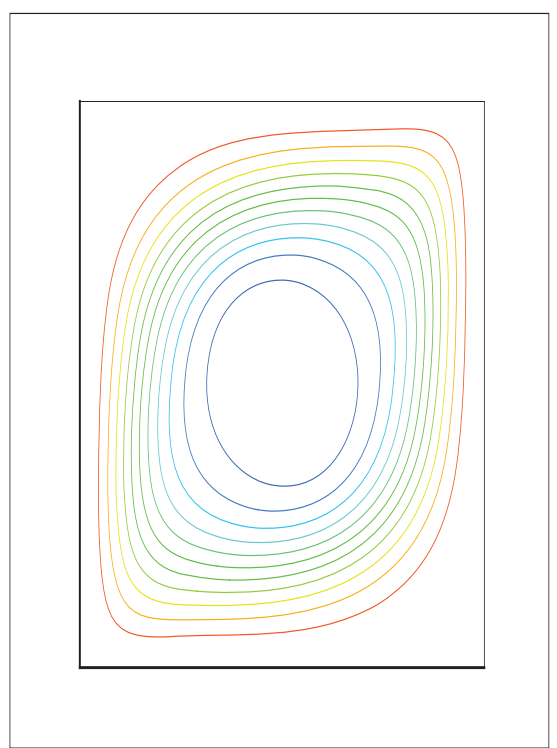

(a)

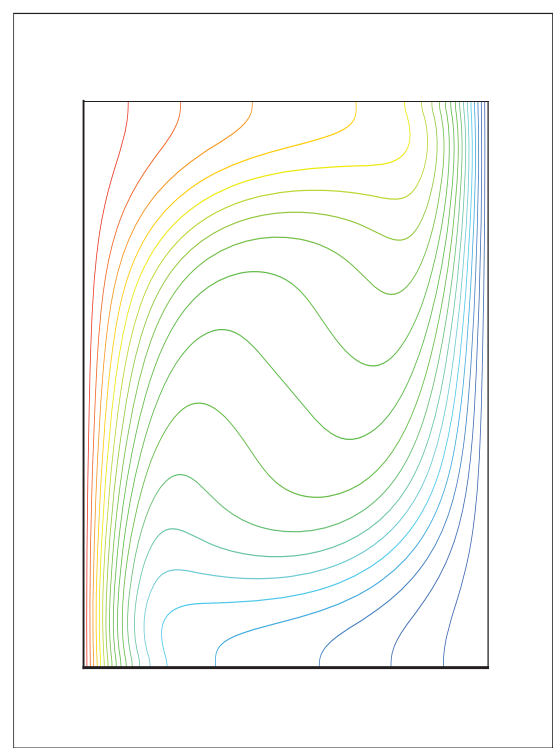

(b)

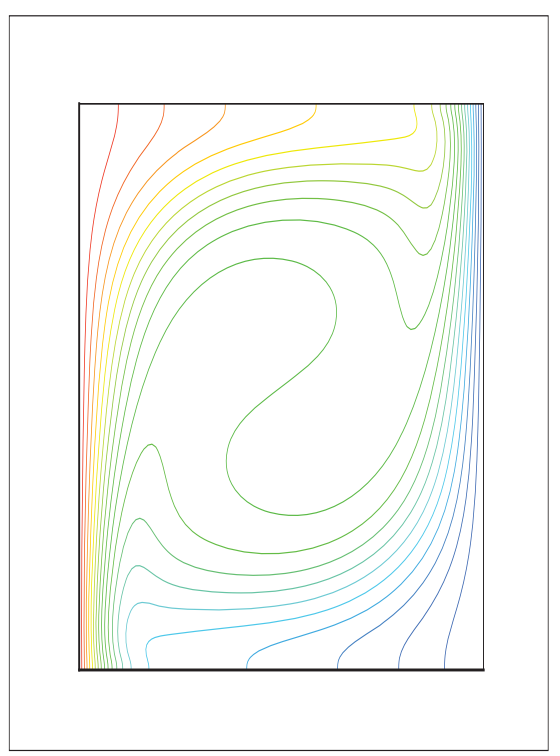

(c)

Fig. 2. (a) streamlines, (b)isotherms and (c) isoconcentrations for $N=-0.8$. 


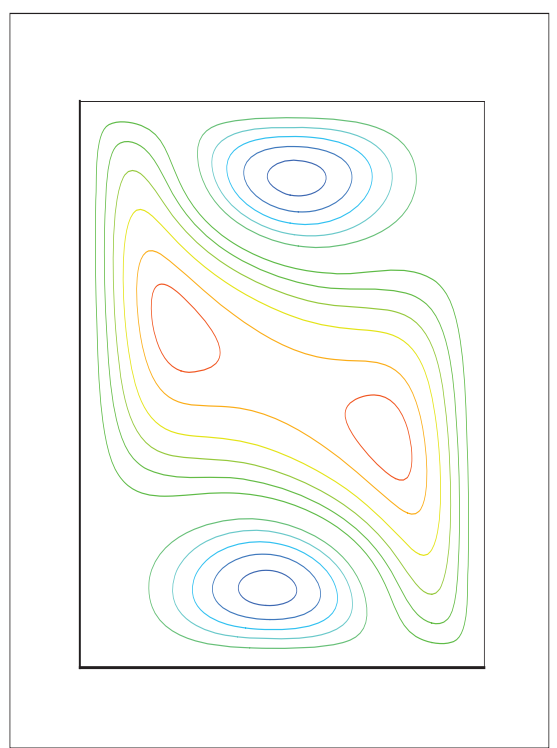

(a)

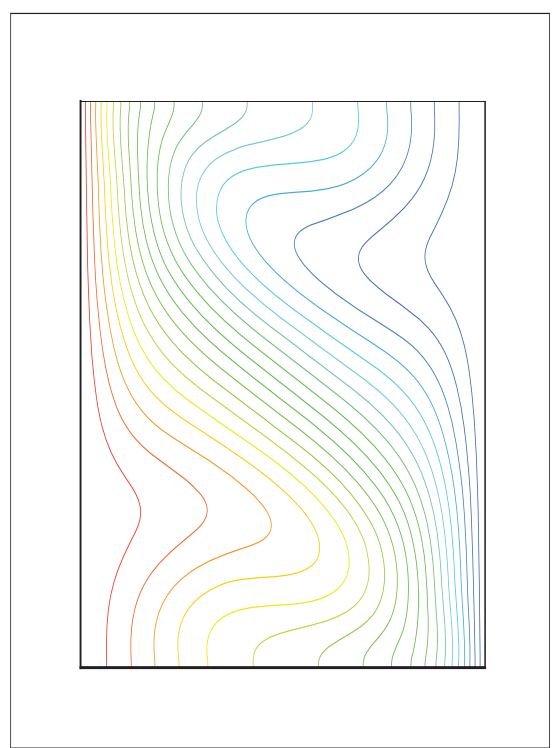

(b)

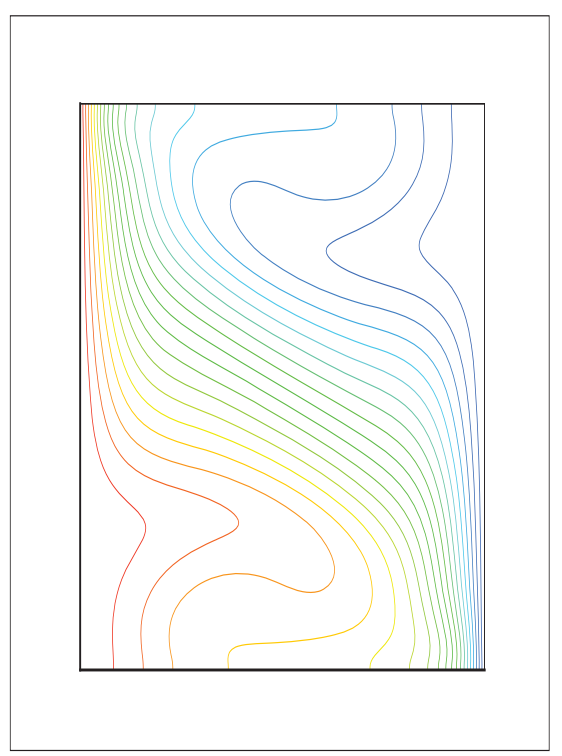

(c)

Fig. 3. (a) streamlines, (b)isotherms and (c) isoconcentrations for $N=-1.3$. 




(a)



(b)

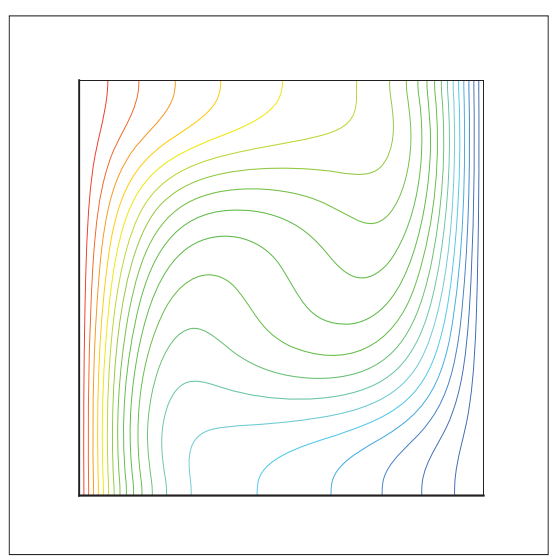

(c)

Fig. 4. (a) streamlines, (b)isotherms and (c) isoconcentrations for $D a=10^{-1}$. 


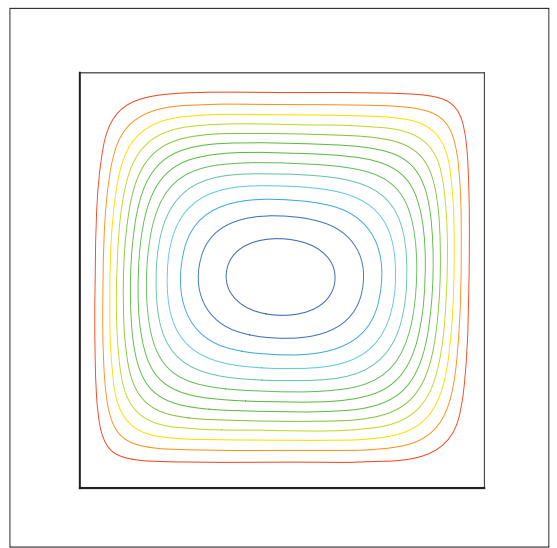

(a)

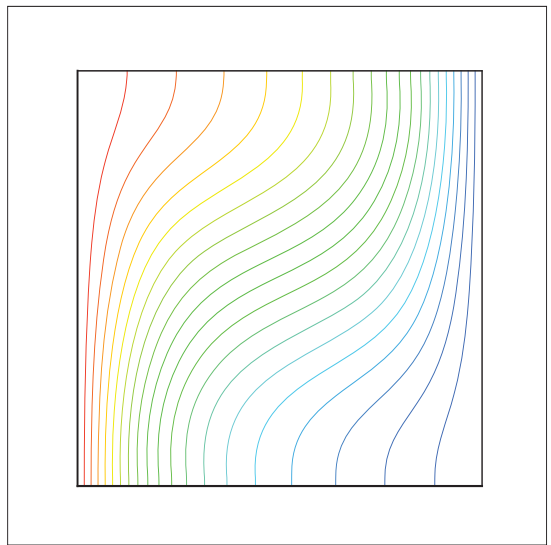

(b)

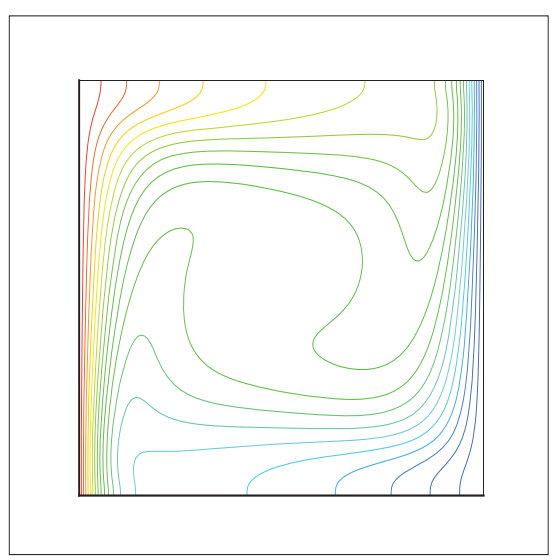

(c)

Fig. 5. (a) streamlines, (b)isotherms and (c) isoconcentrations for $D a=10^{-2}$. 


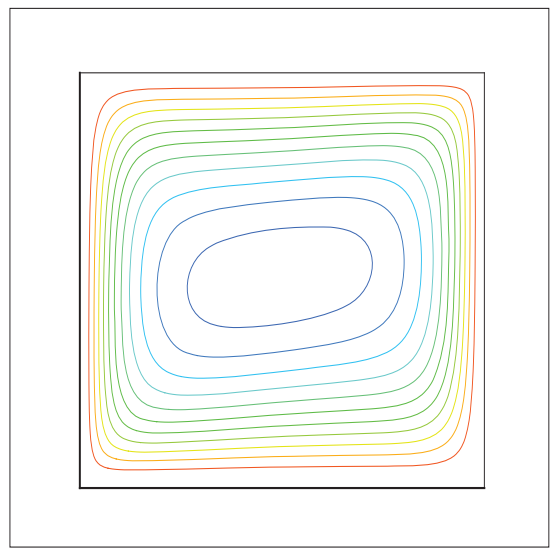

(a)

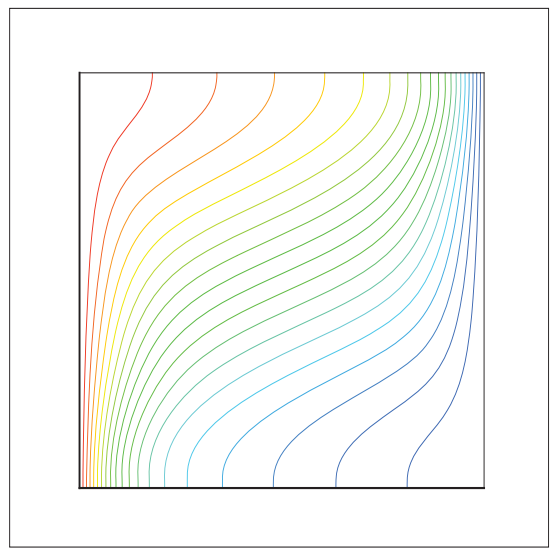

(b)

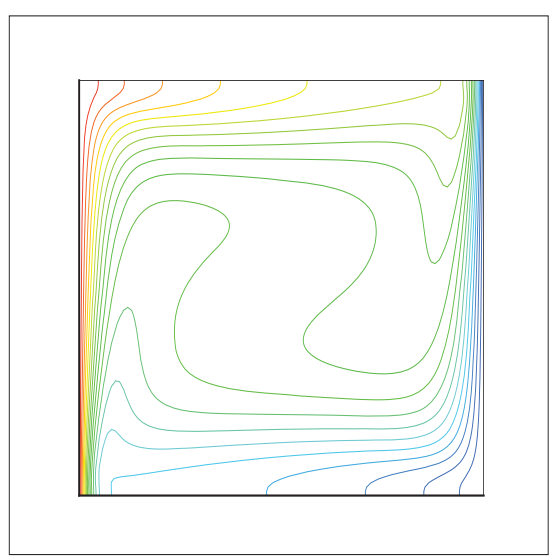

(c)

Fig. 6. (a) streamlines, (b)isotherms and (c) isoconcentrations for $D a=10^{-3}$. 


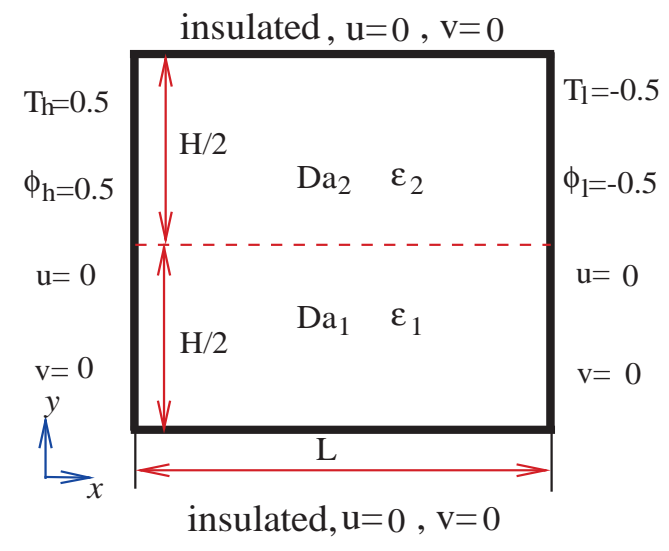

Fig. 7. Schematic configuration of the bi-layered heterogenous porous cavity. 


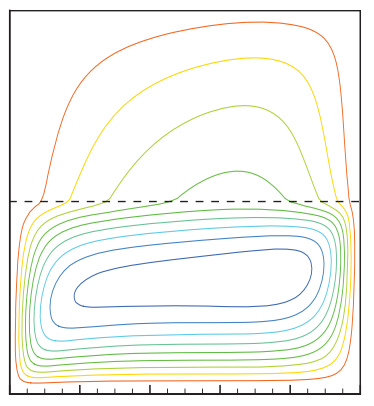

(a)

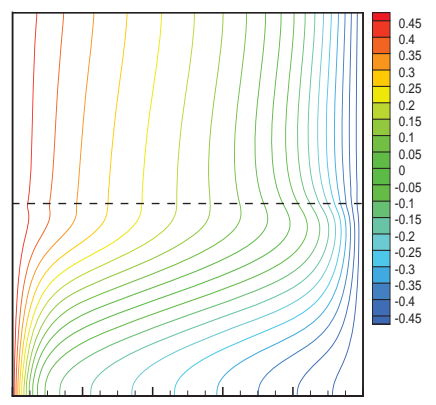

(b)

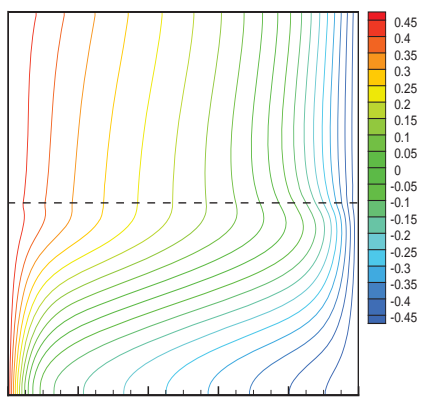

(c)

Fig. 8. (a) streamlines, (b)isotherms and (c) isoconcentrations within the bi-layered heterogenous porous cavity, $L e=1$. 


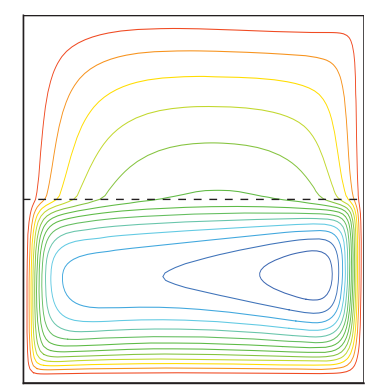

(a)

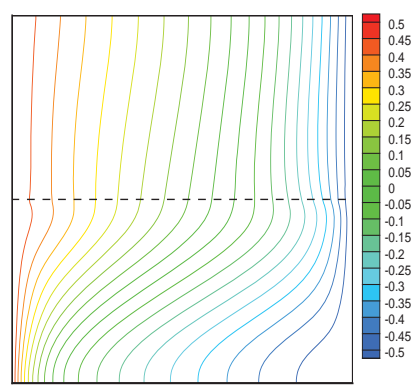

(b)

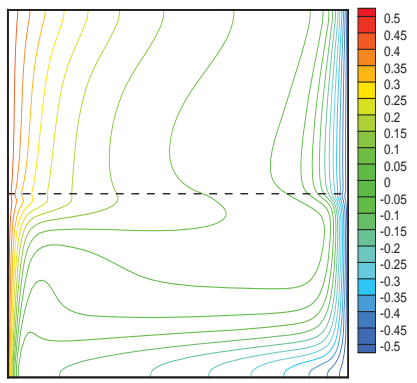

(c)

Fig. 9. (a) streamlines, (b)isotherms and (c) isoconcentrations within the bi-layered heterogenous porous cavity, $L e=10$. 
Table 1

Average Nusselt and Sherwood number on the hot wall.

\begin{tabular}{ccccc} 
& \multicolumn{2}{c}{$N u$} & \multicolumn{2}{c}{ Sh } \\
\hline & Ref.[43] & present & Ref.[43] & present \\
\hline$N=-0.8$ & 3.6897 & 3.6706 & 4.9156 & 4.7576 \\
$N=-1.3$ & 2.1255 & 2.1665 & 3.1615 & 3.1722 \\
\hline
\end{tabular}


Table 2

Average Nusselt number on the hot wall.

\begin{tabular}{cccc}
$D a$ & $10^{-1}$ & $10^{-2}$ & $10^{-3}$ \\
\hline present & 1.094 & 1.730 & 2.567 \\
Ref.[44] & 1.086 & 1.687 & 2.529 \\
\hline
\end{tabular}


Table 3

Average Sherwood number on the hot wall.

\begin{tabular}{cccc}
$D a$ & $10^{-1}$ & $10^{-2}$ & $10^{-3}$ \\
\hline present & 2.936 & 5.695 & 9.712 \\
Ref.[44] & 2.842 & 5.624 & 9.749
\end{tabular}


Table 4

Average Nusselt and Sherwood number on the hot wall.

\begin{tabular}{ccccc} 
& \multicolumn{2}{c}{$N u$} & \multicolumn{2}{c}{$S h$} \\
\hline & Ref.[45] & present & Ref.[45] & present \\
\hline$L e=1$ & 3.37 & 3.27 & 3.37 & 3.28 \\
$L e=10$ & 2.05 & 1.96 & 12.65 & 12.41 \\
\hline
\end{tabular}

